\title{
Improving the reliability and service life of the pneumatic distributor for distributing feed to cages
}

\author{
Evgenii Tihonov ${ }^{1, *}$, Valentin Bazykin ${ }^{2}$, and Nikolay Mukhanov ${ }^{3}$ \\ ${ }^{1}$ Petrosavodsk state university, 33, Lenina st., 185000, Petrozavodsk, Russia \\ ${ }^{2}$ Institute of agroengineering and environmental problems of agricultural production, 3, Filtrovskoe \\ highway, Tyarlevo settlement, 196625, St. Petersburg, Russia \\ ${ }^{3}$ Ivanovo state agricultural academy named after Dmitry Belyaev, 45, Sovetskaya st., 153000, \\ Ivanovo, Russia
}

\begin{abstract}
In this paper, we consider effective delivery of feed to fish cages. The rotary feeders design analysis is performed. Their disadvantages are analyzed. Possible ways to improve reliability are identified: rotor balancing, elimination of moving parts in design. The design of the feeder without moving parts is proposed, which provides the required parameters of feed distribution in cages and at the same time has high reliability and almost unlimited service life. To justify the design parameters of the feeder, we developed design factors that affect the feed distribution process (height of the pipe cut, height of the cone position, radius of curvature of the generatrix), and an integral criterion for evaluating the quality of feed distribution. The integral criterion includes an assessment of the effective area of feed distribution in the cage and its uniformity. A numerical multifactor study was performed and the optimal values of design factors were determined with the maximum value of the integral criterion that determines the efficiency of the feed distribution process. Based on the results of processing the obtained data, the high quality of feed distribution in cages and the prospects of the proposed design of the feeder as a whole were determined.
\end{abstract}

\section{Introduction}

Currently, industrial aquaculture in Russia is experiencing a significant rise. Basically, this segment of the agro-industrial complex is represented by producers of rainbow trout. At the same time, the domestic demand for these products significantly exceeds the supply. Every year, new businesses appear and existing ones expand. Almost every enterprise of industrial aquaculture, in the process of its formation, passes several stages of technological equipment. At the initial stage, while production volumes are relatively small, a significant share in the technological process is occupied by manual labor. This applies to all technological processes of a fish-breeding enterprise: stocking of cages, seating, sorting,

\footnotetext{
*Corresponding author: tihonov@petrsu.karelia.ru
} 
feeding, unloading from cages and shipment. Especially, this is clearly expressed in the feeding process. In small businesses, this process is performed manually. Sometimes, they hand out quite large volumes of feed - up to $500 \mathrm{~kg}$ per cage per day. And there are a lot of cages, even at a small enterprise. In addition to the low efficiency of the use of labor resources (the significance of this indicator depends very much on the cost of labor in the region), a big question is the quality of feed distribution and control of compliance with the requirements of the main fish breeder of the enterprise.

When certain production volumes are reached, almost any enterprise moves to the next stage of mechanization of the feeding process. This stage is characterized by the use of mobile pneumatic feeders of various designs: from fairly small (loading capacity 2001 .) to relatively large (loading capacity $1200-1500$ l.) [1]. When using feeders with large loading volumes, there is a problem with the dosage. As a rule, these feed dispensers do not have dosing systems, which leads to a decrease in the accuracy of delivery and, as a result, a decrease in the overall efficiency of feeding. There are two possible options: underfeed and, as a result, a decrease in the daily growth of biomass, and overfeed-the fish will not eat all the food, and it will fall to the bottom of the reservoir under the cage. In the second case, the feed coefficient will increase. The most advanced design of a mobile feed dispensers have a dozing system. For example, a feed feeder developed in the Engineering Park of the Institute of forest mining and construction Sciences of Petrozavodsk state University [2]. This feeder is equipped with a dispenser for $25 \mathrm{~kg}$. This is very convenient, since the most common packaging of fish food is $25 \mathrm{~kg}$. in view of this, often the main fish breeders of enterprises round the back feeding rate to a multiple of $25 \mathrm{~kg}$.

As production increases, the use of mobile feed distribution systems becomes inefficient. Large feeding volumes require a large number of flights from the loading point to the feeding point. This forces enterprises to move to the next stage of mechanization of the feeding process - automation. This stage involves the use of stationary (water-based) feeding systems that provide full automation [3]. These systems automatically determine the feeding rate and feed coefficient depending on the water temperature, oxygen concentration, and average fish weight in the cage. They also take into account daily biomass growth and waste. Such kind of systems can provide maximum efficiency of feed by use fine tuning of feeding process $[4,5]$. As a rule, one such system serves up to 16 cages and has a maximum capacity of up to $500 \mathrm{~kg} / \mathrm{h}$. Only limitation is the length of pneumatic feed lines. It should not exceed $300 \mathrm{~m}$.

The most vulnerable point of automatic feed systems is the point of direct feed delivery to the cage. Sometimes, the company can limit itself to fixing the feed pipe on the rail of the cage. In this case, the spot where the feed falls into the cage will be spindly with a small area. The effectiveness of feeding will be reduced. To ensure the most effective spot of feed falling into the cage, rotary distributors are used, the so-called "spreaders" [6] (see figure $1)$. 


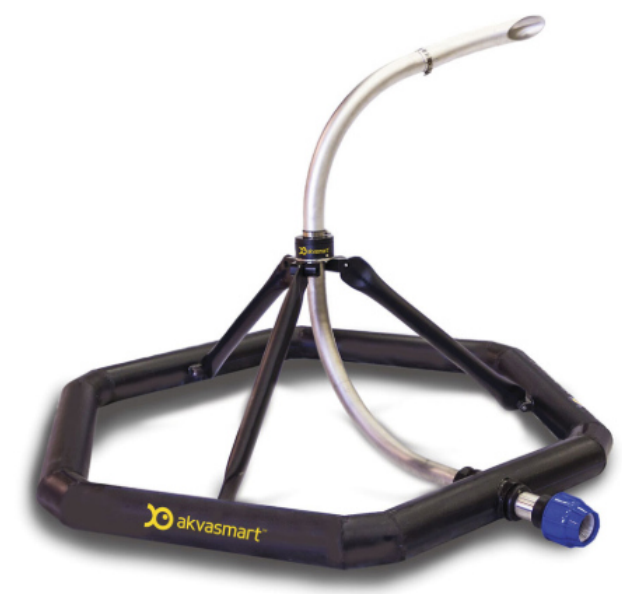

Fig. 1. Pneumatic rotary feed distributors [6].

These pneumatic feed distributors provide a feed drop spot in the form of a ring with an outer diameter slightly smaller than the inner diameter of the cage, and the smallest possible inner diameter (see figure 2).

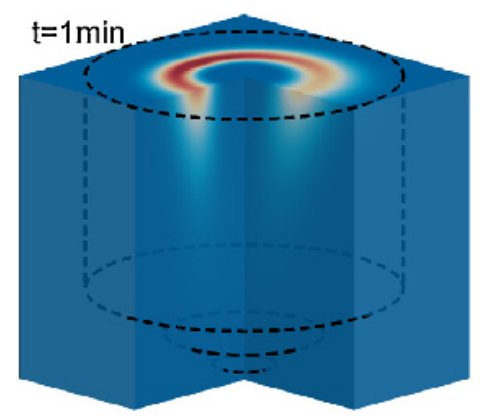

Fig. 2. Distribution of feed inside the cage when distributed by a rotary distributor [7].

These feeders provide maximum feeding efficiency, but are not without disadvantages. The first is the absence of domestic analogues and the high price (up to 200000 rubles). The second is insufficient uniformity of feed distribution from the center to the edge of the cage [7]. There is a sufficient amount of research on this subject in the world [8,9], which shows that the design of rotary distributors is close to optimal, and it is not possible to improve their characteristics without using new approaches. As can be seen from figure 3, effective feed distribution is made on an area with a diameter of up to $18 \mathrm{~m}$, while the size of cages for commercial fish reaches $30 \mathrm{~m}$ in diameter. 


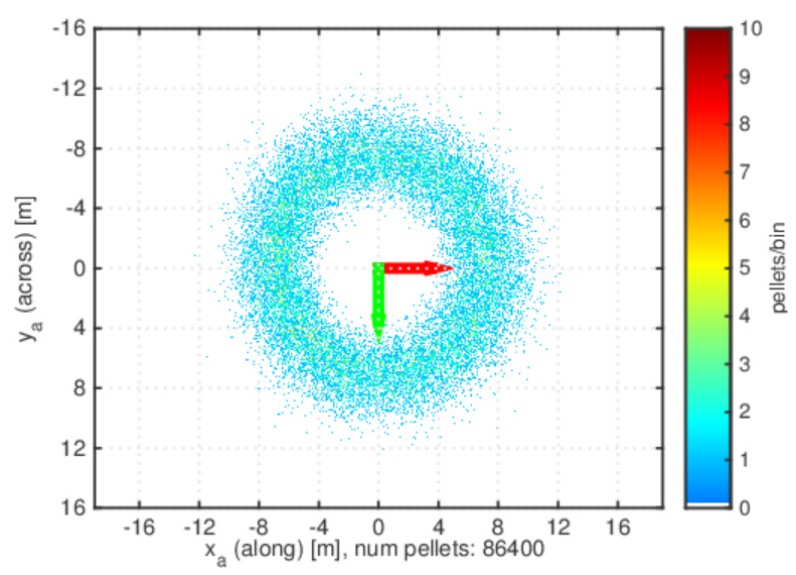

Fig. 3. Distribution of feed in the cage [7].

Based on this, it turns out that the area of effective distribution of feed is about $250 \mathrm{~m} 2$, while the total area of the cage is about $700 \mathrm{~m}^{2}$. Over $400 \mathrm{~m}^{2}$ is not used.

The second drawback is related to the design and principle of operation of the device. The feeder has a curved feed line, ending with a hinged, curved branch pipe. This branch pipe rotates, thanks to a jet of air supplied under pressure from the feed pipe. This ensures a circular feed delivery to the cage. The weak point of the structure is the hinge (see figure 4).

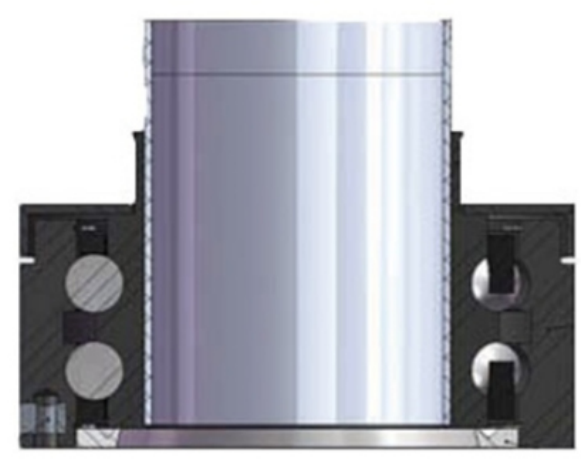

Fig. 4. Hinge joint of the rotating branch pipe [6].

Since the structure of the joint is exposed to moisture, it is made in the form of a bearing made of polymer materials. The rotating part of the branch pipe is not balanced, so the inner ring of the bearing is affected by a cyclic bending moment, which significantly reduces the service life of this feed feeder unit. As practice shows, the life of this bearing is approximately 1,5 years. If the node fails, the rotating part of the pipe is jammed, and the area of feed delivery is reduced to a small spot in one part of the cage.

The danger of this situation is that the failure may not be detected immediately, which will lead to a significant decrease in the efficiency of feeding the cage. Also, the company must have a stock of bearings, since it will be impossible to organize an operational delivery due to the lack of domestic analogues. However, the most unfavorable type of failure is the separation of the rotating part of the pipe and its fall to the bottom of the cage. In this situation, the feed will be fed vertically up, which in the presence of wind will lead to displacement of the spot of falling feed from the cage and underfeed the fish. Also, it will 
be possible to lift the torn part of the pipe only with the help of divers or when lifting the pipe, which happens infrequently.

In this regard, it is necessary to solve the problem of reliability of rotary feeders and within the framework of the import substitution program to offer the domestic industry a technical solution of a feed feeder with an "unlimited resource" and high reliability.

\section{Materials and methods}

In the process of solving this problem, the following methods were applied: patent search, theory of solving inventive problems, numerical modeling of multiphase gas dynamic systems, multi-criteria optimization.

The patent search was performed in the search engine of the Federal Institute of industrial property for the following queries: feed distributor, feed distributor, feed distribution system. More than 50 patent documents were analyzed. The analysis showed the absence of any useful models, inventions or methods that provide an "unlimited resource" and high reliability of feed distribution to the cage with the required parameters.

To increase the bearing life, possible ways to change the design of the rotating part of the branch pipe were analyzed. The most effective condition for increasing the service life is to eliminate the cyclic bending moment acting on the inner ring of the bearing. This can be achieved by applying static balancing of the rotating part of the pipe using a counterweight. The offset and weight of the counterweight must be selected so that the center of mass is on the axis of rotation of the pipe (see figure 5).

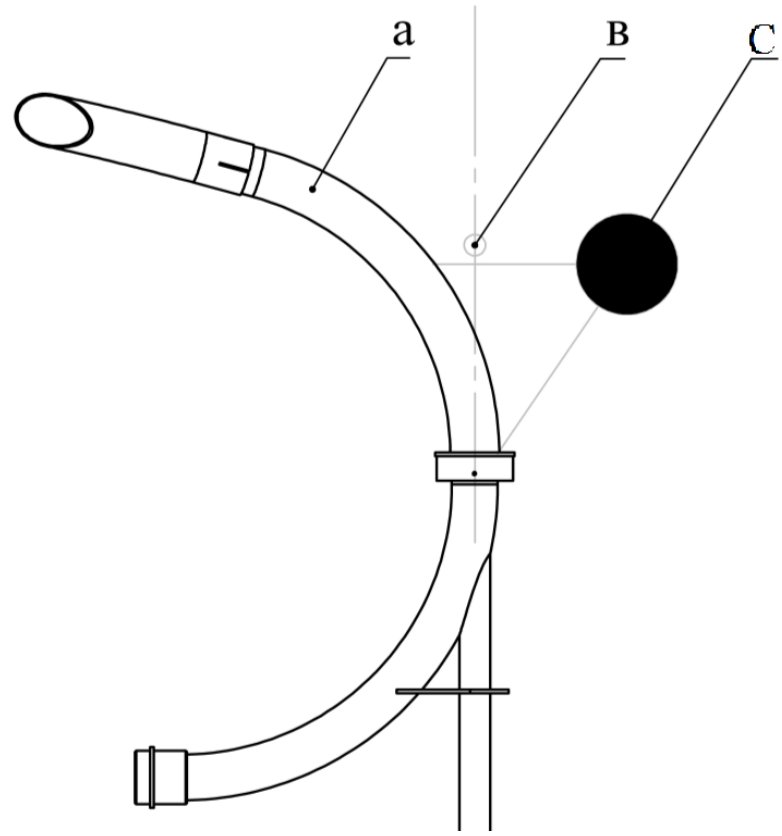

Fig. 5. - Rotating branch pipe of a rotary feed distributor with a counterweight: a - rotating branch pipe; $b$ - counterweight; $c$ - center of mass.

At the same time, the resource can be significantly increased, but it is not possible to predict failure. However, it is known from the course of General reliability theory that the reliability of a system is inversely proportional to the number of kinematic pairs in the 
system. In this regard, it is necessary to apply a technical solution that excludes kinematic pairs in the device.

Analysis of domestic research on this topic has shown a complete lack of publications of any developments and results on this topic. Foreign researchers pay attention to rotary feeders. But their research is usually concerned with the dynamics of the working process, the uniformity of feed distribution, and the issues of forced fluctuations that occur during work $[10,11]$. Research conducted abroad does not apply to the design of a rotary feeder, its reliability and resource. It seems that the current technical solution for distributing feed is considered optimal.

As a scheme that excludes moving parts in the design of the feed feeder, Petrozavodsk state University has developed a schematic diagram shown in figure 6.

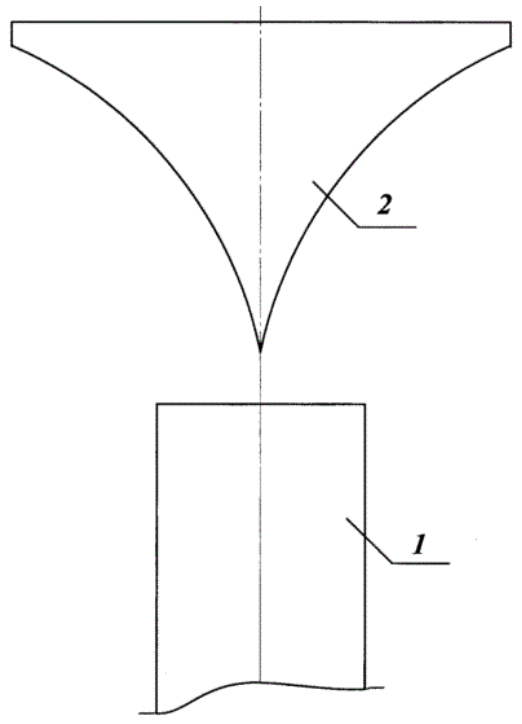

Fig. 6. Concept of a feed distributor without moving parts: 1 - feeding pipe ; 2 - reverse cone [12].

This scheme works as follows: the feed, flying out of the vertical branch of the pneumatic feed pipe 1 , deviates at a certain angle, hitting the reverse cone 2 . In this case, the feed deflection will be axisymmetric. This idea is protected by the RU184230 patent [12].

The main question that arose when working out this design is the shape of the reverse cone generatrix. The shape of the reverse cone should ensure the maximum possible uniformity of distribution of feed from the center of the cage to its edge. For a preliminary estimation of the distribution, we performed a simulation of the feed distribution process in the flow simulation numerical simulation environment, a SolidWorks software package. Such systems are widely used for modeling gas-dynamic processes of pneumatic transport of bulk substances $[13,14,15]$. Visualization of simulation results is shown in figure 7. 


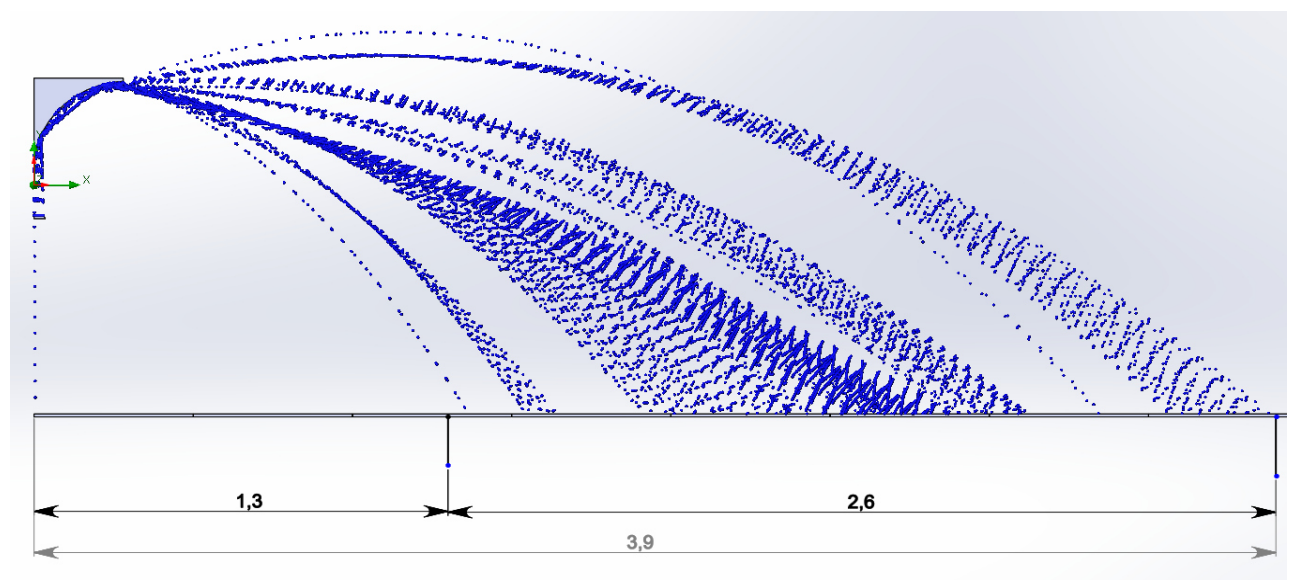

Fig. 7. Visualization of preliminary modeling results.

As you can see from the figure, the feed begins to fall into the water at a distance of 1,3 $\mathrm{m}$ from the feeder. Further, the specific mass of feed falling per unit area increases, reaching a certain maximum, and then the mass decreases and decreases to 0 at a distance of $3,9 \mathrm{~m}$ from the distributor. The distribution of the amount of feed depending on the distance from the feeder is shown in figure 8 .

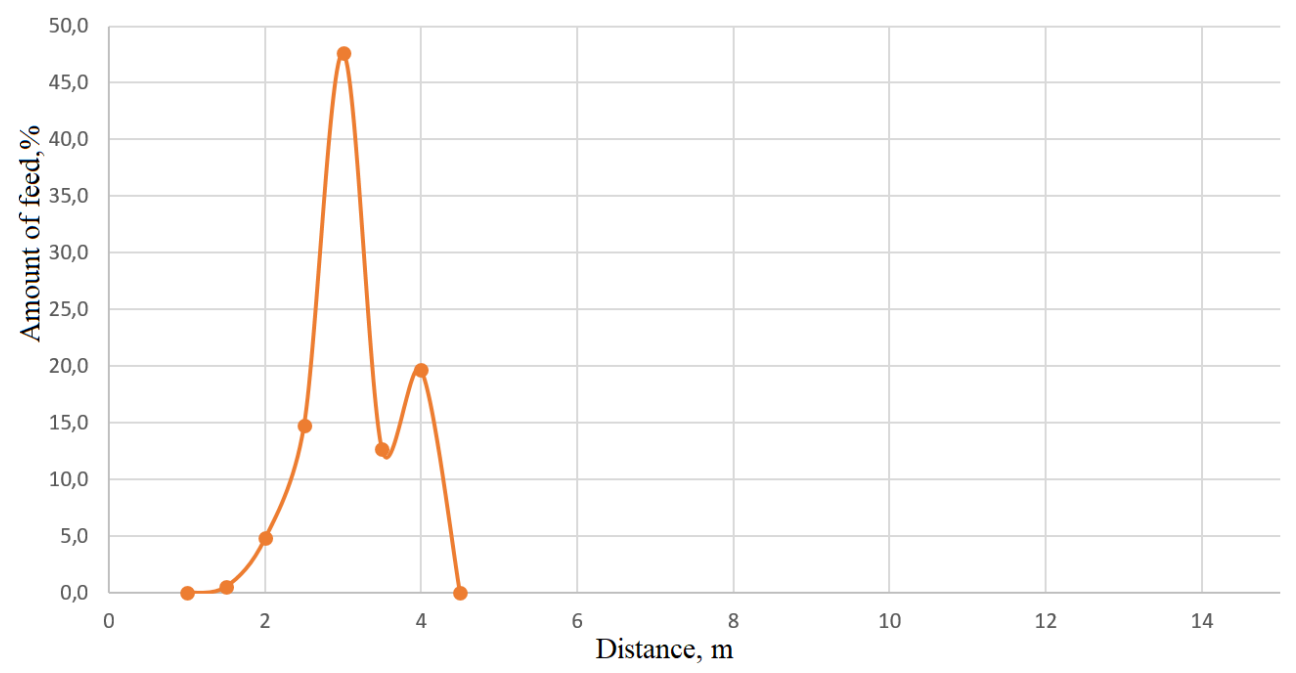

Fig. 8. Distribution of feed by cage radius.

Preliminary modeling showed the efficiency of the proposed technical solution and the need to increase the range of feed ejection and achieve maximum uniformity of distribution over the radius of the cage.

Since the largest diameter of cages used in Russia, as a rule, does not exceed $30 \mathrm{~m}$, then, ideally, it is necessary to ensure the area of feed falling into the cage in the form of a ring with an outer diameter of $25 \mathrm{~m}$ and an inner diameter of $0 \ldots .5 \mathrm{~m}$. These parameters will be optimization criteria for further multi - factor research.

To achieve this goal, a number of studies were performed on the influence of the design parameters of the distributor on the range and uniformity of feed distribution. The simulation result is shown in figure 9. 


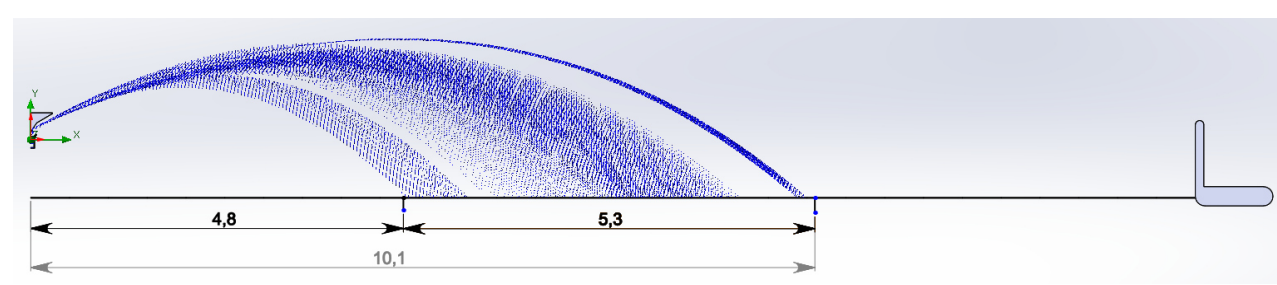

Fig. 9. Visualization of simulation results.

The feed distribution is shown in figure 10.

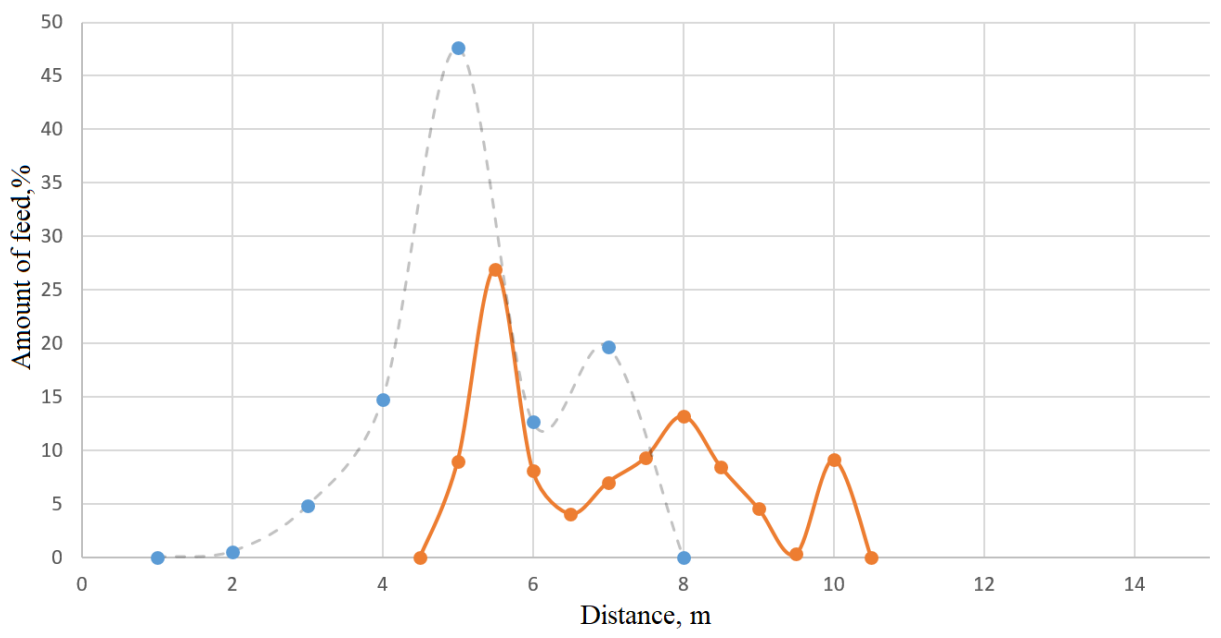

Fig. 10. Distribution of feed by cage radius: the dashed line represents the distribution of the feed shown in figure 8 .

Let's define the geometric parameters of the distributor as factors that affect the optimization criteria (see figure 11).

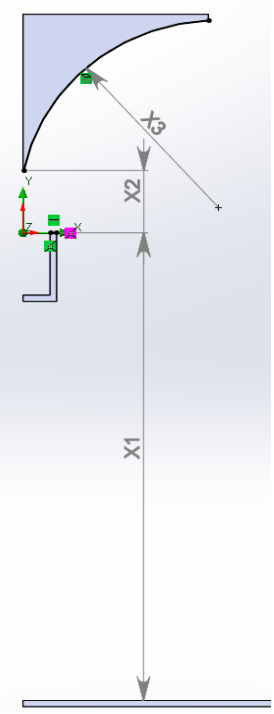

Fig. 11. Geometric parameters of the distributor. 
The factors and their varying levels are presented in table 1.

Table 1. Factors and their varying levels.

\begin{tabular}{|c|l|c|c|c|}
\hline \multirow{2}{*}{ № } & \multicolumn{1}{|c|}{ Factor } & \multicolumn{3}{|c|}{ Varying levels } \\
\cline { 3 - 5 } & & $\mathbf{- 1}$ & $\mathbf{0}$ & $+\mathbf{1}$ \\
\hline $\mathrm{X}_{1}$ & Cutting height of the branch pipe, $\mathrm{mm}$ & 1000 & 1500 & 2000 \\
\hline $\mathrm{X}_{2}$ & Height of the cone position, mm & 0 & 50 & 100 \\
\hline $\mathrm{X}_{3}$ & Radius of curvature of the cone generatrix, $\mathrm{mm}$ & 150 & 250 & 350 \\
\hline
\end{tabular}

When determining the level of variation in the height of the pipe section and the height of the cone position, the results of preliminary modeling were taken into account. With the radius of generatrix curvature the situation is somewhat more complex.

The simulation results show that the part of the feed pellets coming out of the pipe closer to its axis collides with the surface of the cone at a small angle. Then, reflecting at the same angle, it collides with the upper part of the cone (see figure 12).

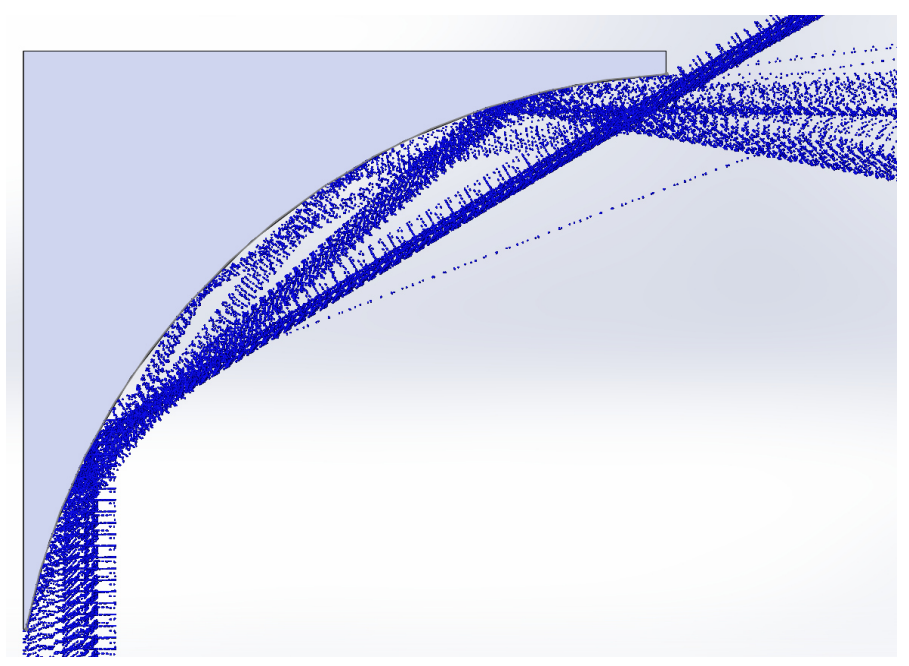

Fig. 12. Reflections of feed pellets from the surface of the cone generatrix.

Pellets flying out of the pipe closer to its walls hit the middle part of the cone surface and deflect at a sufficient angle to avoid a second collision. From these collisions, some of the pellets can be destroyed. On the one hand, the smaller the collision angle, the smaller the impact on the pellet. On the other hand, if the angle is small, the granule will collide with the cone generatrix part 2 times. Moreover, some of the pellets can be destroyed by such dynamic loads [16].

The most important aspect of collisions is the kinetic energy of the pellet, which will decrease, passing into deformation and heating, thereby reducing the range of the pellet until it falls into the water. As a result, the $\mathrm{X}_{3}$ factor will affect the parameters of the feed distribution in the cage.

The height of the cone position $\mathrm{X}_{2}$ will also affect the feed distribution parameters, since the pellets will have an initial spread when they leave the nozzle. And the higher the position of the cone, the greater the spread.

As optimization criteria, we define the outer radius of the ring of the feed drop site-Y1 and the width of the ring $-\mathrm{Y}_{2}$. Also, it is necessary to take into account the factor of uniformity of distribution of the amount of feed given out over the width of the ring-S1. This criterion is defined as follows. The $Y_{2}$ value is divided into 10 sections: from $Y_{2-1}$ to 
$\mathrm{Y}_{2-10}$. Next, the ratio of the area with the maximum amount of falling feed to the area with the minimum amount is estimated:

$$
S_{1}=\frac{Y_{2-m u x}}{Y_{2-m i n}}
$$

\section{Results}

According to the developed research plan, a number of numerical experiments were performed. First, the results were analyzed for satisfaction of the $Y_{1}$ criterion. Experiments showing a value of $Y_{1}<9 \mathrm{~m}$ were excluded from further analysis. Further analysis was based on the following statements: $\mathrm{Y}_{2}$ should tend to the maximum, and the criterion $\mathrm{S}_{1}-$ to the minimum. From this we derive the integral optimization criterion:

$$
Y S-\frac{Y_{2}}{S_{1}} \rightarrow M A X
$$

Visualization of simulation results is shown in figure 13.

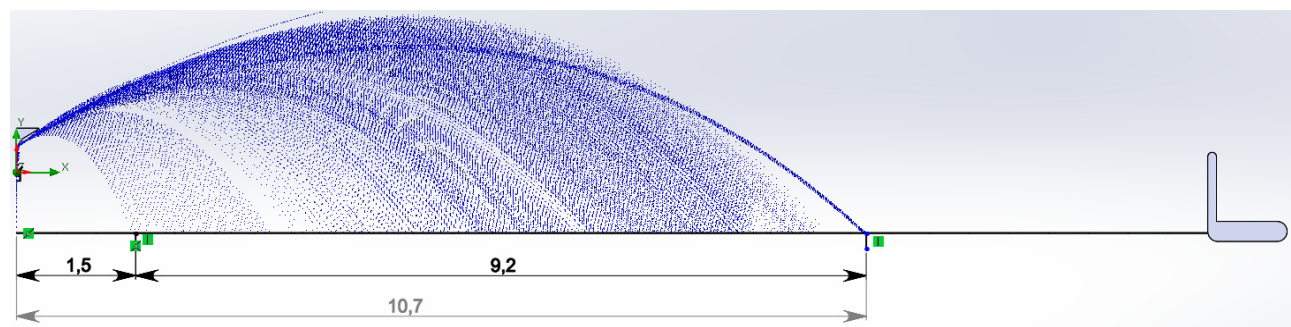

Fig. 13. Simulation of feed distribution with the maximum value of the integral factor YS.

The feed distribution is shown in figure 14.

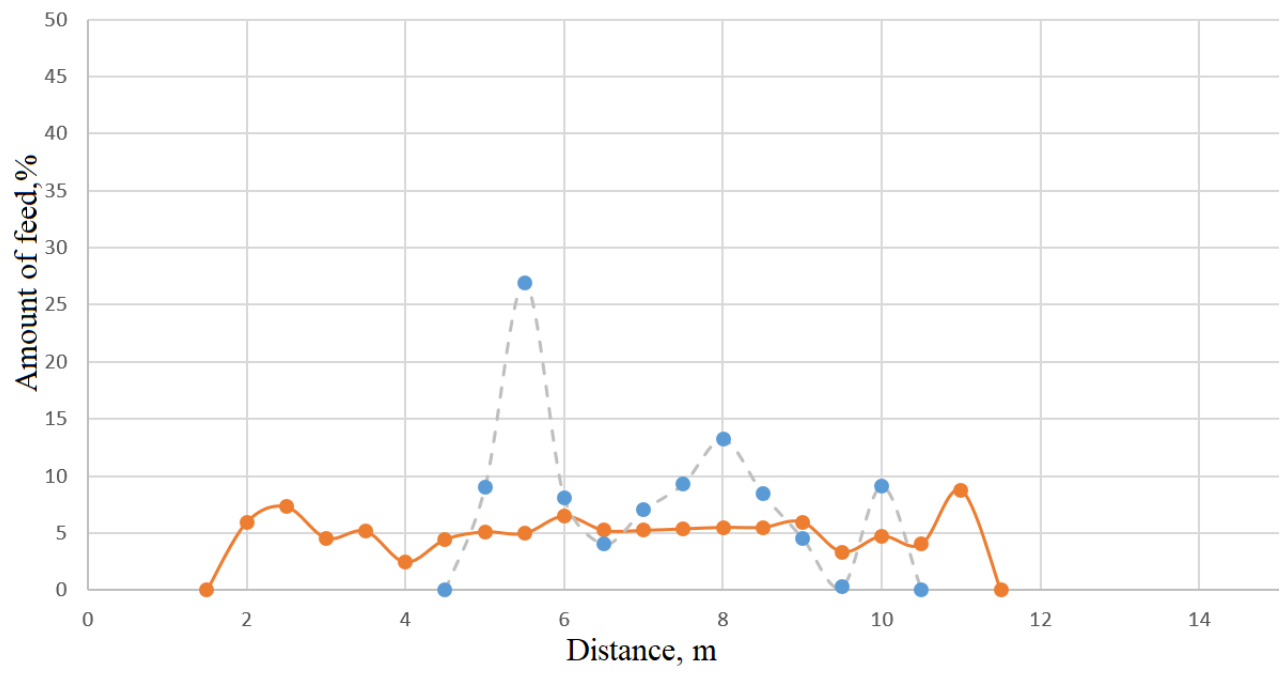

Fig. 14. Distribution of feed by cage radius: the dashed line represents the distribution of the feed shown in figure 10. 
The values of the achieved criteria are as follows:

$\mathrm{Y}_{1}=10,7 \mathrm{~m}$;

$\mathrm{Y}_{2}=9,2 \mathrm{~m}$;

$\mathrm{S}_{1}=3,57$;

$\mathrm{YS}=2,58$.

The values of factors that provide the maximum integral optimization criterion is shown in table 2 .

Table 2. Values of factors that provide $\mathrm{YS}=\max$.

\begin{tabular}{|c|l|c|}
\hline № & \multicolumn{1}{|c|}{ Factor } & Value \\
\hline$X_{1}$ & Cutting height of the branch pipe, $\mathrm{mm}$ & 1610 \\
\hline $\mathrm{X}_{2}$ & Height of the cone position, $\mathrm{mm}$ & 75 \\
\hline $\mathrm{X}_{3}$ & Radius of curvature of the cone generatrix, $\mathrm{mm}$ & 345 \\
\hline
\end{tabular}

It should be noted that the calculation does not take into account the interaction between feed pellets in flight. In reality, the uniformity of feed distribution will be higher due to collisions between pellets.

\section{Discussion and conclusion}

The research has shown the principal possibility and technical feasibility of the technical solution of the feeder, which provides "unlimited resource", high reliability and uniformity of feed distribution with maximum use of the internal area of the cage. "Unlimited resource" is a relative concept. In the proposed technical solution, there are no moving parts, and the only part subject to attrition is the cone. The circular generatrix of the cone will gradually deteriorate into collision with the pellet feed. However, the resource of this part will be several orders higher than that of the bearing, which is used in existing rotary distributors. At the same time, the rejection of the proposed design will be a change in the parameters of the feed drop site in the cage. These changes will occur gradually and can be diagnosed by the company's engineering staff during scheduled maintenance of cages. A worn cone can be easily replaced with a new one. At the same time, the design of the distributor is completely import-substituting.

\section{References}

1. IAS Products Ltd. All rights reserved https://iasproducts.com/products/aerospreader-s500-feed-broadcaster

2. Feeders. Petrozavodsk state university. Engineering park https://petrsu.ru/structure/6906/pages/8284/kormorazdattchiki

3. Automatic feeding systems. Petrozavodsk state university. Engineering park (2020) https:/petrsu.ru/structure/6906/pages/8265/avtomatitcheskie-kormovye-sistemy

4. A. Salgado-Ismodes, S. Taipale, J. Pirhonen, Aquaculture 519 (2020) https://doi.org/10.1016/j.aquaculture.2019.734919

5. N. Papandroulakis, P. Dimitris, D. Pascal, Aquacultural Engineering 26(1), 13-26 (2012) https://doi.org/10.1016/S0144-8609(01)00091-7

6. Akva group. Rotor spreaders (2019) https://www.akvagroup.com/pen-basedaquaculture/feed-systems/rotor-spreaders/

7. M. Oehme, T. Synnøve Aas, Mette Pe, I. Lygren, T. Åsgård, Aquacultural Engineering 51, 44-52 (2012) doi.org/10.1016/j.aquaeng.2012.07.001 
8. K.R. Skøien, J.A. Alfredsen, Oceans - St. John's, 1-7 (2014) doi: 10.1109/OCEANS.2014.7003103

9. M.O. Alver, K. Rist Skøien, M. Føre, T. Synnøve Aas, M. Oehme, J. Arve Alfredsen, Aquacultural Engineering 72-73, 20-29 (2016) https://doi.org/10.1016/j.aquaeng.2016.03.003

10. K. Rist Skøien, M. Omholt Alver, J. Arve Alfredsen, Aquacultural Engineering 82, 111 (2018) https://doi.org/10.1016/j.aquaeng.2017.11.002

11. K. Rist Skøien, M. Omholt Alver, A. Piotr Zolich, J. Arve Alfredsen, Computers and Electronics in Agriculture https://doi.org/10.1016/j.compag.2016.08.020

12. Federal institute of industrial property (FIIP) https://www.fips.ru/iiss/search_res.xhtml?faces-redirect=true

13. H. Liu, D. Du, P. Shen, B. Chang, W. Zhang, J. Pan, Annals of Nuclear Energy 111, 568-578 (2018) https://doi.org/10.1016/j.anucene.2017.09.009

14. Z. Miao, S. Kuang, H. Zughbi, A. Yu, Powder Technology 361, 62-73 (2020) https://doi.org/10.1016/j.powtec.2019.10.057

15. T. Synnøve Aas, M. Oehme, M. Sørensen, G. He, I. Lygren, T. Åsgård, Aquacultural Engineering 44(91), 25-34 (2011) https://doi.org/10.1016/j.aquaeng.2010.11.002 